\title{
A New Fast Algorithm for Image Denoising
}

\author{
Shixiu Zheng ${ }^{1}$, Zhenkuan Pan , Chunxiao Jiang, Guodong Wang
}

\begin{abstract}
In this paper, the classic TV-L1 model is extended to a hybrid TV-L1 model based on first order and second order derivatives to remove image noises, preserve geometry, contrast and overcome staircase effects. To improve efficiency, a fast algorithm based on dual method which is called the Split-Dual algorithm is designed for the model. Finally, the denoising quality and the computational time between the proposed algorithm and some other traditional methods are compared.
\end{abstract}

Keywords: variational method; image denoising; TV-L1 model; Dual method; Split-Dual algorithm

\section{Introduction}

The variational models of diffusion using first order derivative can efficiently remove image noises with edge preserving property. The TV(Total Variation) model or ROF model proposed by Rudin, Osher and Fatemi[1] is very popular in image processing and has been considered as one of the most important nonlinear variational image diffusion models. The TV-L1 model was proposed by Allinet[2] for digital signal filters in 1992, then it was extended to impulsive noise removal of images and developed by the researchers in the area of image processing due to its property of geometry preserving. They are models both based on first order derivative and they usually lead to staircase effects.

To preserve geometry, contrast and overcome staircase effects, researchers mixed regularizers using first order and second order derivatives. Didas, Weickert, Burgeth[3], Pan, Wei and Zhang[4] analyzed the corresponding 1D model Which can be extended to $2 \mathrm{D}$ cases directly and made the following conclusion as

$$
\left\{\begin{array}{cc}
\text { First order forward diffusion } & \Phi_{1}<0 \\
\text { No first order forward diffusion } & \Phi_{1}=0 \\
\text { First order backward diffusion } & \Phi_{1}>0
\end{array}\right.
$$

\footnotetext{
${ }^{1}$ Shixiu Zheng $(\bowtie)$

College of Information Engineering, Qingdao University, 266071 ,China.

e-mail: zhengwang_001@163.com
} 


$$
\left\{\begin{array}{cc}
\text { Second order backward diffusion } & \Phi_{2}<0 \\
\text { No second order forward diffusion } & \Phi_{2}=0 \\
\text { Second order forward diffusion } & \Phi_{2}>0
\end{array}\right.
$$

Where $\Phi_{1}\left(u_{x x}^{2}\right)=2 g^{\prime \prime}\left(u_{x x}^{2}\right) u_{x x}^{2}+3 g^{\prime}\left(u_{x x}^{2}\right) \quad, \quad \Phi_{2}\left(u_{x x}^{2}\right)=2 g^{\prime}\left(u_{x x}^{2}\right) u_{x x}^{2}+g\left(u_{x x}^{2}\right)$ ， $g\left(u_{x x}^{2}\right)=\varphi^{\prime}\left(\left|u_{x x}\right|\right) /\left|u_{x x}\right|$.

But the method based on traditional gradient descent equations to solve these models is complicated, Goldstein, Osher[5]propose the Split Bregman algorithm based on the work of Osher, Burger[6]and Wang, Yang, Yin, Zhang[7]. Auxiliary variables and Bregman iterative parameters are introduced and the soft thresholding formulas are used to transform the calculation into alternative minimization. In recent years, the Dual method[8-10] has been developed by the researchers in the area of image processing. In this method, dual variables are introduced to avoid the complex curvature term and enhance the efficiency of computing.

In this paper, a variational model via convex combination of regularizers based on first and second derivatives is proposed along with its fast algorithm. The proposed hybrid models can remove both gauss noise and salt-pepper noise efficiently. Finally, the denoising quality and the computational time between the proposed algorithm and some other traditional methods are compared.

The paper is organized as follows: A hybrid TV-L1 model based on first order and second order derivatives is proposed in section 2 along with its dual method. The improved Split-Dual method for the model is proposed in section 3. Some numerical examples are given in section 4 to compare the effect of the proposed algorithm with some other traditional methods. Conclusions are given finally.

\section{The hybrid TV-L1 model based on first order and second order derivatives and its Dual method}

An observed scalar value image $f(x), x \in \Omega$ with additive noise can be considered as the sum of clear image $u$ and noise $\xi: f=u+\xi$. The classic TV-L2 model[1] has some disadvantages about contrast loss and geometry loss of images ,these can be improved efficiently by TV-L1 model with L1 data term. The classic TV-L1 model can be stated as the following minimization problem of energy function[2]

$$
E(u)=\lambda \int_{\Omega}|\nabla u| d x d y+\int_{\Omega}|u-f| d x d y
$$

To preserve geometry, contrast and overcome staircase effects, hybrid regularizers using first order and second order derivatives are proposed in this paper for TV-L1 model based on the work in [4] and [11] with the following energy functional 


$$
\underset{u}{\operatorname{Min}}\left\{E(u)=\int_{\Omega}|u-f| d x+\lambda \int_{\Omega}|\nabla u| d x+\beta \int_{\Omega}|\Delta u| d x\right\}
$$

The method based on traditional gradient descent equations to solve this model is

$$
\frac{\partial u}{\partial t}=-\frac{u-f}{|u-f|}+\nabla\left(\frac{\nabla u}{|\nabla u|}\right)-\Delta\left(\frac{\Delta u}{|\Delta u|}\right)
$$

It is a fourth order partial differential equation and its finite difference scheme is quite complex with low computational efficiency even using semi-implicit iterative schemes.

In order to improve computational efficiency, a fast algorithm which is called dual method is designed for it firstly. Three dual variables $p_{1}, p_{2}, p_{3}$ are introduced in this method, then the data term, the total variation term and the high order term are transformed into the following forms

$$
\begin{aligned}
& \int_{\Omega}|u-f| d x=\operatorname{Sup}_{p_{1}:\left|p_{1}\right| \leq 1} \int_{\Omega}(u-f) p_{1} d x \\
& \int_{\Omega}|\nabla u| d x=\underset{\underset{p_{2}}{\mathrm{r}}:\left|p_{2}\right| \leq 1}{\operatorname{Sup}} \int_{\Omega} u \nabla \cdot \stackrel{\mathrm{r}}{p_{2}} d x \\
& \int_{\Omega}|\Delta u| d x=\operatorname{Sup}_{p_{3}:\left|p_{3}\right| \leq 1} \int_{\Omega} u \Delta p_{3} d x
\end{aligned}
$$

So model(4) is transformed into such problem of maximum and minimum as follows

$\underset{u}{\operatorname{Min}} \underset{p_{1}:\left|p_{1}\right| \leq 1}{\operatorname{Sup}} \operatorname{Sup}_{p_{2}:\left|p_{2}\right| \leq 1} \operatorname{Sup}_{p_{3}:\left|p_{3}\right| \leq 1}\left\{E\left(u, p_{1}, \stackrel{\mathrm{r}}{p_{2}}, p_{3}\right)=\int_{\Omega}(u-f) p_{1} d x+\lambda \int_{\Omega} u \nabla \cdot \stackrel{\mathrm{r}}{p_{2}} d x+\beta \int_{\Omega} u \Delta p_{3} d x\right\}$

Firstly, $p_{1}, \stackrel{1}{p}_{2}, p_{3}$ are fixed based on the alternative minimization, then the EulerLagrange equation on $u$ can be derived easily as follows

$$
\frac{\partial E}{\partial u}=p_{1}+\lambda \nabla \cdot p_{2}+\beta \Delta p_{3}
$$

Based on explicit difference scheme of gradient descent, the equation on $u$ can be derived as follows

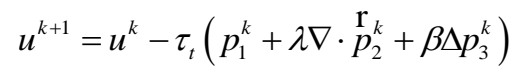

Based on Semi-implicit difference scheme and KKT condition (Karush-KuhTucker)[9], $p_{1}, \stackrel{1}{p}_{2}, p_{3}$ are calculated by equations as follows

$$
\begin{gathered}
p_{1}^{k+1}=\frac{p_{1}^{k}+\tau_{p_{1}}\left(u^{k+1}-f\right)}{1+\tau_{p_{1}}\left|u^{k+1}-f\right|} \\
{\stackrel{\mathrm{p}}{p_{2}^{k+1}}}^{k}=\frac{\stackrel{p}{2}_{2}^{k}-\tau_{p_{2}} \nabla u^{k+1}}{1+\tau_{p_{2}}\left|\nabla u^{k+1}\right|}
\end{gathered}
$$




$$
p_{3}^{k+1}=\frac{p_{3}^{k}+\tau_{p_{3}} \Delta u^{k+1}}{1+\tau_{p_{3}}\left|\Delta u^{k+1}\right|}
$$

Where, $\tau$ is the time step, $\tau_{t} \tau_{p_{1}}<1 / 8, \tau_{t} \tau_{p_{2}}<1 / 8, \tau_{t} \tau_{p_{3}}<1 / 8[9]$.

\section{The new fast algorithm- -Split-Dual algorithm}

The dual method proposed in section 2 works very fast, and it is much faster than the Split Bregman algorithm. But it leads to blurring in some images. So a hybrid algorithm is proposed based on the algorithm in this section, which is called the Split-Dual algorithm. Auxiliary variable $s$ and dual variables $\stackrel{1}{p}_{2}, p_{3}$ are introduced, so model (4) is transformed into such problem of maximum and minimum as follows

$$
\operatorname{Min}_{u, s} \operatorname{Sup}_{p_{2}:\left|p_{2}\right| \leq 1} \operatorname{Sup}_{p_{3}:\left|p_{3}\right| \leq 1}\left\{\begin{array}{l}
E\left(u, s, \stackrel{\mathrm{r}}{p_{2}}, p_{3}\right)=\int_{\Omega}|s| d x+\lambda \int_{\Omega} u \nabla \cdot \stackrel{\mathrm{r}}{p_{2}} d x+ \\
\beta \int_{\Omega} u \Delta p_{3} d x+\frac{\vartheta}{2} \int_{\Omega}(s+u-f)^{2} d x
\end{array}\right\}
$$

The difference between (9) and (13) is the processing about the data term. In (9), dual variables are introduced to transform such problem into maximum and minimum, then $u$ is calculate based on explicit difference scheme of gradient descent. In (13), split variable $s$ is introduced, then the Euler-Lagrange equation on $u$ and the analytic solution on $u$ can be derived as follows

$$
\begin{gathered}
\vartheta(s+u-f)+\lambda \nabla \cdot \stackrel{\mathrm{p}}{2}_{2}+\beta \Delta p_{3}=0 \\
u^{k+1}=f-s^{k}-\frac{1}{\theta}\left(\lambda \nabla \cdot \stackrel{\mathrm{r}}{k}_{2}^{k}+\beta \Delta p_{3}^{k}\right)
\end{gathered}
$$

By the soft thresholding formulas, the analytic solution on $s$ can be derived as follows

$$
s^{k+1}=\operatorname{Max}\left(\left|f-u^{k+1}\right|-\frac{1}{\theta}, 0\right) \frac{f-u^{k+1}}{\left|f-u^{k+1}\right|}
$$

$\stackrel{1}{p}_{2}, p_{3}$ are the same as (12.2)and(12.3).

The algorithm can be summarized as:

(a) Initialize $k=0, s^{0}=p_{2}{ }^{0}=p_{3}{ }^{0}=0, u^{0}=f$.

(b) While $\frac{\left|E^{(k+1)}-E^{(k)}\right|}{\left|E^{(k)}\right|} \geq \varepsilon$, do

(1) Calculate $u^{k+1}$ using (15); (2) Calculate $s^{k+1}$ using (16); (3) Calculate $p_{2}{ }^{k+1}$ using (12.2); (4) Calculate $p_{3}{ }^{k+1}$ using (12.3); (5) $k \rightarrow k+1$, goto (b).

(c) End while. 


\section{Numerical examples}

The purpose of the numerical example is to test the denoising quality and computational efficiency between the proposed model and some other methods. All the experiments are implemented on a PC computer (Pentium(R) Dual-Core CPU G620 2.60GHz, Memory 3.40GB) using Matlab7.3.0. The computing equation of SNR for scalar images is $S N R=10 \cdot \log _{10}\left(\frac{\int_{\Omega}(f-\bar{f})^{2} d x d y}{\int_{\Omega}(n-\bar{n})^{2} d x d y}\right)$.

Where $f$ is the original image, $\bar{f}$ is the average of the original image. $n$ is the noise, $\bar{n}$ is the average of the noise.

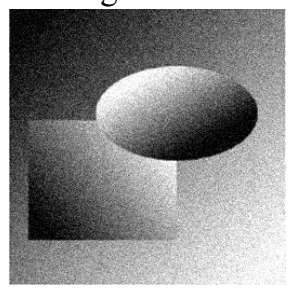

(a) Image with Gauss noise

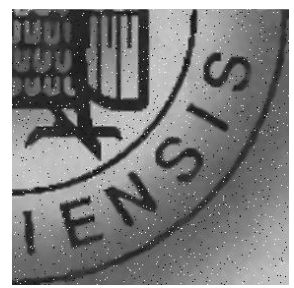

(b) Image with salt and pepper noise

Fig. 1 The original images and the noisy images

Fig.1 (a) shows a scalar image of $256 * 256$ which is corrupted by white Gauss noise with standard deviation $\sigma=20$. Fig. 1 (b) shows a scalar image of $256 * 256$ which corrupted by salt and pepper noise with the density $\mathrm{d}=0.05$.

To compare the efficiency of different algorithms, four kinds of experiences are made in this paper. One is traditional gradient descent equations method(GDE). Another is Split Bregman algorithm(SBA). The third is dual method proposed in section 2(DM). The fourth is the new fast algorithm, Split-Dual algorithm(SDA) proposed in section 3 .

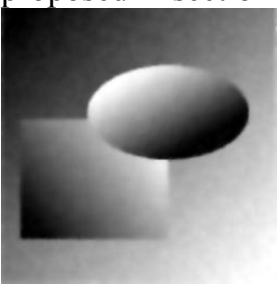

(a)

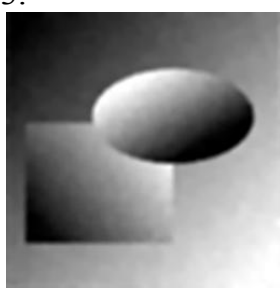

(b)

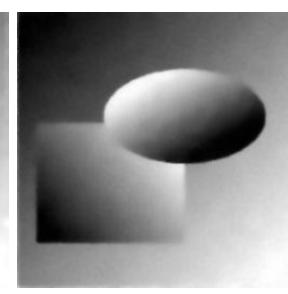

(c)

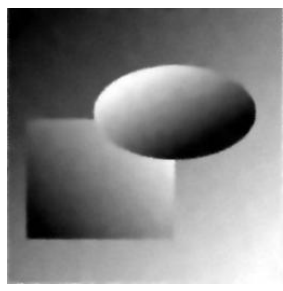

(d)

Fig. 2 Compare of Gauss noise removal with four algorithms. (a) Result using gradient descent equations (b)Result using Split Bregman algorithm (c) Result using Dual method (d) Result using Split-Dual algorithm 
Fig. 2 shows the denoising of Gauss noise. Fig.2 (a) shows the image restoration using traditional gradient descent equations for the proposed model, penalty parameters are $\lambda=20, \beta=20, \Delta t=0.01$. Fig. 2 (b) shows the image restoration using

Split Bregman algorithm, penalty parameters are $\lambda=20, \beta=10, \theta_{1}=10, \theta_{2}=1, \theta_{3}=0.5, \theta_{4}=1$. Fig.2 (c) shows the image restoration using Dual method designed in section 2, penalty parameters are $\tau=1 / 8, \lambda=30, \beta=10$. Fig. 2 (d) shows the image restoration using Split-Dual algorithm designed in section 3 , penalty parameters are $\tau=1 / 8, \lambda=16, \beta=9, \theta=9$.

The proposed model can remove not only gauss noise, but also salt and pepper noise effeciently.

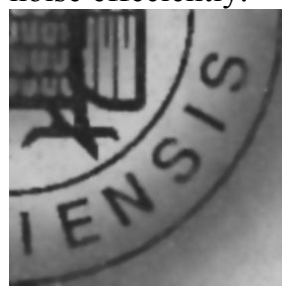

(a)

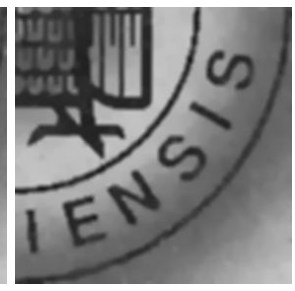

(b)

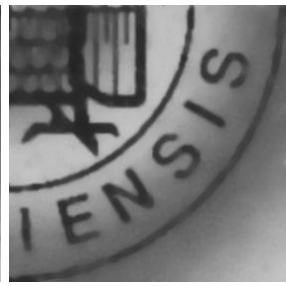

(c)

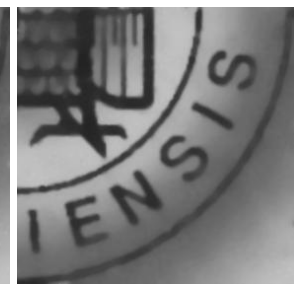

(d)

Fig.3 Compare of salt and pepper noise removal with four algorithms. (a) Result using gradient descent equations (b) Result using Split Bregman algorithm (c) Result using Dual method (d) Result using Split-Dual algorithm

Fig.3 shows the denoising of salt and pepper noise. Fig.3 (a) shows the image restoration using gradient descent equations for the proposed model, penalty parameters are $\lambda=20, \beta=20, \Delta t=0.01$. Fig. 3 (b) shows the image restoration using Split Bregman algorithm, penalty parameters are $\lambda=20, \beta=10, \theta_{1}=8, \theta_{2}=1, \theta_{3}=0.5, \theta_{4}=1$. Fig. 3 (c) shows the image restoration using Dual method designed in section 2, penalty parameters are $\tau=1 / 8, \lambda=25, \beta=10$. Fig. 3 (d) shows the image restoration using Split-Dual algorithm designed in section 3 , penalty parameters are $\tau=1 / 8, \lambda=19, \beta=10, \theta=10$.

To compare the efficiency of different algorithms, energy decreasing charts of four kinds of algorithms are drawn together. From the trend of the curves, it is obviously that the proposed Split-Dual algorithm make energy decrease the fastest(the thick line). 


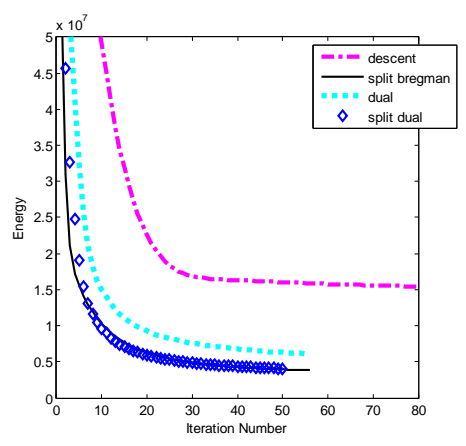

(a) Corresponding energy curve for Fig.2

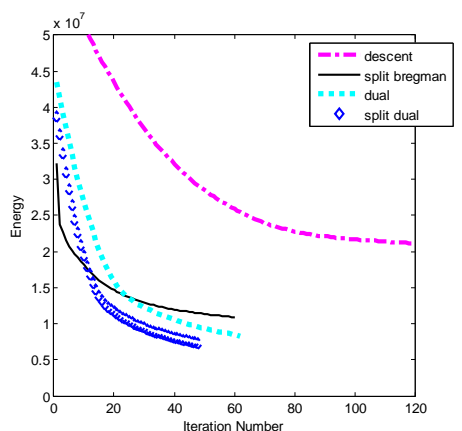

(b) Corresponding energy curve for Fig.3

Fig.4 Energy decreasing charts

The SNR(Signal to Noise Ratio) and the computing costs are listed in Table 1 for comparison. The original SNR in Figure 1(a) is 10.8634, in Figure 1(b) is 7.9119.

Table 1 SNR and computing time

\begin{tabular}{ccccc}
\hline \multirow{2}{*}{ Algorithms } & $\begin{array}{c}\text { SNR } \\
\text { Fig.1(a) }\end{array}$ & $\begin{array}{c}\text { SNR } \\
\text { Fig.1(b) }\end{array}$ & $\begin{array}{c}\text { CPU time(s) } \\
\text { Fig.1(a) }\end{array}$ & $\begin{array}{c}\text { CPU time(s) } \\
\text { Fig.1(b) }\end{array}$ \\
\hline \multirow{2}{*}{ GDE } & 19.9461 & 13.9872 & 6.2810 & 6.9840 \\
SBA & 18.4266 & 14.6596 & 4.7350 & 3.6560 \\
DM & 20.5211 & 13.5741 & 0.3440 & 0.5320 \\
SDA & 21.6669 & 14.6401 & 0.4070 & 0.6870
\end{tabular}

The experiments show that the Split Bregman algorithm is faster than the method based on gradient descent equations, and the Dual method is much more faster than the Split Bregman algorithm, but sometime images will be bluring, the SNR is lower than the Split Bregman algorithm. The hybrid Split-Dual algorithm takes the advantages of the two fast algorithms. The computing efficiency and the SNR are both improved by a large margin. The proposed algorithm can remove both gauss noise and salt-pepper noise.

\section{Conclusions}

The Dual method and the Split-Dual algorithm are designed for the hybrid TV L1 model in this paper. Experiments show that the proposed algorithm is easy to implement and has high computation efficiency compared with classic methods. But our algorithm is not very effective for noisy images with texture, so models using non-local means will be researched in the future. 


\section{Acknowledgment}

This work was supported by National Natural Science Foundation of China (No.6 1305045 and No.61170106) and Qingdao science and technology development pr oject (No. 13-1-4-190-jch).

\section{References}

1. L. Rudin, S. Osher and E. Fatemi, Nonlinear Total Variation Based Noise Removal Algorithms [J], Physica D, 60:259-268, 1992

2. S.Alliney, Digital filters as absolute norm regularizers, IEEE Transactions on Signal Processing, 40(6), pp. 1548-1562, 1992.

3. S. Didas, J. Weickert, B. Burgeth, Properties of Higher Order Nonlinear Diffusion Filtering, J Math Imaging Vis, 35(3):208-226, 2009

4. Z. Pan, W. Wei, H. Zhang, The Variational Image Difussion Model Using Gradient and Laplacian, Journal of Shandong University(Edition of Sciences), 43(11):11-16, 2008

5. T. Goldstein, Osher S. The Split Bregman Method for L1 Regularized Problems, SIAM Journal on Imaging Sciences, Volume 2 Issue 2, April 2009, Pages 323-343

6. S. Osher, M. Burger, D. Goldfarb, J. Xu, W. Yin. An iterative regularization method for total variation based on image restoration[J]. Multiscale Modeling and Simulation ,20054 (2):460489

7. Y. Wang, J. Yang, W. Yin and Y. Zhang, A New Alternating Minimization Algorithm for Total Variation Image Reconstruction, SIAM Journal on Imaging Sciences (2008), Volume: 1, Issue: 3, Publisher: Citeseer, Pages: 248-272

8. Chambolle A., An algorithm for total variation minimization and applications, Journal of Mathematical Imaging and Vision, 20(1):89-97, 2004.

9. Brown E. S., Chan T. F., Bresson X., Completely convex formulation of the Chan-Vese image segmentation model, International Journal of Computer Vision, 98(1):103-121 , 2012.

10. Wu C., Tai X.-C., Augmented Lagrangian method, dual methods, and Split Bregman iteration for ROF, vectorial TV, and high order models, SIAM Journal on Imaging Sciences, 3(3):300-339, 2010

11. S. Zheng, Z. Pan, G. Wang, A Variational Model of Image Restoration Based on First and Second Order Derivatives and Its Split Bregman Algorithm, ICALIP 2012, 860-865, 2012. 